\title{
On the Scattering of the Plane Electromagnetic Wave by a Continuous Inhomogeneity with Spherical Symmetry in a Homogeneous Medium
}

\author{
by \\ K. Naito \\ Meteorological Reseach Institute
}

(Received December 11, 1952)

\begin{abstract}
By applying the wave-mechanical treatments in atomic collisions the author investigates the scattering of the plane electromagnetic waves by a continuous inhomogeneity with spherical symmetry in a homogeneous medium. An example case is given for such a spatial distribution of an inhomogeneity in a medium as $\exp \left(-R^{2} / R_{0}^{2}\right)$.
\end{abstract}

\section{Introduction}

In the recently developed radiometeorology, the influence of rain-drops on microwave propagation has been treated according to the theory of the scattering of plane electromagnetic waves by a sphere, which is due to Mie and Stratton. In a similar manner we may discuss the influence of atmospheric turbulence on microwave propagation according to the theory of the scattering of plane electromagnetic waves by a continuous inhomogeneity with spherical symmetry in a homogeneous medium. Here we shall treat such a scattering problem.

In an inhomogeneous, isotropic and nonconductive medium without free charge density, where the spatial variation of its characteristics may be considered to be continuous and gradual, a vector $\vec{C}$ representing either electric field $\vec{E}$ or magnetic field $\vec{H}$ satisfies the following wave equation:

$$
\vec{\nabla}^{2} C-\varepsilon \mu(x, y, z) \frac{\partial^{2} \vec{C}}{\partial t^{2}}=0, *
$$

where $\varepsilon$ and $\mu$ denote permitivity and permeability in the medium respectively. If the vector $\vec{C}$ contains $e^{-i \omega t}$ as the time factor, equation (1) becomes

$$
\left\{\begin{array}{l}
\nabla^{2} \vec{C}+k^{2}(y, y, z) \vec{C}=0, \\
k^{n}=\varepsilon \mu(x, y, z) \omega^{2} .
\end{array}\right.
$$

* Equation (1) is valid only when the gradients of medium characteristics are small. Therefore it should be noticed that equation (1) is meaningless beyond the limit. 
The solutions of equation (2), expressed in spherical coordinates $(R, \theta, \phi)$, can be constructed from the vectors $\vec{M}$ and $\vec{N}$ which are derived from the solutions of the scalar wave equation

$$
\nabla^{2} \varphi+k^{2}(x, y, z) \phi=0
$$

in the following manner:

$$
\left\{\begin{array}{l}
\vec{M}=\nabla \varphi \times \vec{R}=\frac{1}{k} \nabla \times \vec{N}, \\
\vec{N}=\frac{1}{k} \nabla \times \vec{M} . *
\end{array}\right.
$$

Here the position vector $\vec{R}$ is equal to $\overrightarrow{i_{R}} R$. It is clear that $\vec{M}$ and $\vec{N}$ are proper functions to represent $\vec{E}$ and $\vec{H}$ under the conditions postulated in the derivation of equation (1), because each one is proportional to the rotation of the other and both have divergence equal to zero as can easily be shown. We shall express $\vec{E}$ and $\vec{H}$ in the form

$$
\left\{\begin{array}{l}
\vec{E}=\sum_{n}\left(p_{n} \vec{M}_{n}+q_{n} \vec{N}_{n}\right), \\
\vec{H}=\frac{k}{i \omega \mu} \sum_{n n}\left(p_{n} \vec{N}_{n}+q_{n} \vec{M}_{n}\right),
\end{array}\right.
$$

where the coefficients $p_{n}$ and $q_{n}$ are to be determined from boundary conditions.

\section{Integration of vector wave equation}

In order to solve the vector wave equation (2) for a plane wave arriving at an inhomogeneity in a medium, we must, first of all, obtain the solutions of the scalar wave equation (3).

Now we shall treat such an inhomogeneity as follows:

$$
\left\{\begin{array}{l}
\varepsilon \mu=\varepsilon_{0} \mu_{0}(\text { constant })+k^{\prime}(R) / \omega^{2}, \\
k^{2}=k_{0}{ }^{2}+k^{\prime}(R), \\
k_{0}{ }^{2}=\varepsilon_{0} \mu_{0} \omega^{2}, \\
k^{\prime}(R) \rightarrow 0 \quad \text { as } \quad R \rightarrow \infty,
\end{array}\right.
$$

where the center of the inhomogeneity is chosen to be the origin of the coordinates.

Equation (3) then becomes

$$
\nabla^{2} \mathscr{P}+k_{0}^{2} \mathcal{P}=-k^{\prime} \mathscr{P} .
$$

Without inhomogeneity, i.e., $k^{\prime}=0$, equation (7) is reduced to

$$
\nabla^{2} \dot{\rho}+k_{0}^{2} \varphi=0 \text {. }
$$

The solutions of equation (8) must be valid in constructing of an incident plane wave, without an inhomogeneity in a medium. Then the solutions of equation (8) are expressed by

$$
\mathscr{P}_{e^{m n}}=j_{n}\left(k_{0} R\right) P_{n}^{m}(\cos \theta) \underset{\sin }{\cos } m \phi e^{-i \omega t}[1]
$$

\footnotetext{
* Considering the conditions postulated in the derivation of equation (1), see Reference [1].
} 
Using the generating functions of equation (9), the incident plane wave, whose electric vector is linearly polarized in the $x$-direction and whose propagation is directed to the positive $z$-axis, is expressed in the form

$$
\begin{aligned}
& \left\{\begin{array}{l}
\vec{E}=\overrightarrow{a_{x}} E_{0} e^{i k_{0} z-i \omega t}=E_{0} e^{-i \omega t} \sum_{n=1}^{\infty} i^{n} \frac{2 n+1}{n(n+1)}\left(\vec{m}_{01 n}-i \vec{n}_{e 1 n}\right), \\
\vec{H}=\overrightarrow{a_{y}} \frac{k_{0}}{\mu_{0} \omega} E_{0} e^{i k_{0} z-i \omega t}=-\frac{k_{0} E}{\mu_{0} \omega} e^{-i \omega t} \sum_{n=1}^{\infty} i^{n} \frac{2 n+1}{n(n+1)}\left(\vec{m}_{c 1 n}+i \overrightarrow{n_{01 n}}\right), \\
\vec{m}_{0_{1 n}}= \pm \sin \theta \\
e_{n}\left(k_{0} R\right) P_{n}{ }^{1}(\cos ) \underset{\sin }{\cos } \phi \overrightarrow{i_{\theta}}-j_{n}\left(k_{0} R\right) \frac{\partial P_{n}{ }^{1} \sin }{\partial \theta} \overrightarrow{\cos } \phi \overrightarrow{i_{\phi}},
\end{array}\right. \\
& \overrightarrow{n_{0_{0}}}=\frac{n(n+1)}{k_{0} R} j_{n}\left(k_{0} R\right) P_{n}{ }^{1}(\cos \theta) \frac{\sin }{\cos } \phi \overrightarrow{i_{R}}+\frac{1}{k_{0} R}\left[k_{0} R j_{n}\left(k_{0} R\right)\right]^{\prime} \frac{\partial P_{n}{ }^{1} \sin }{\partial \theta} \cos \phi \overrightarrow{i_{\theta}} \\
& \pm \frac{1}{k_{0} R \sin \theta}\left[k_{0} R j_{n}\left(k_{0} R\right)\right]^{\prime} P_{n}{ }^{1}(\cos \theta) \underset{\sin }{\cos } \phi \overrightarrow{i_{\phi}} \text {. }
\end{aligned}
$$

Here $\overrightarrow{a_{x}}, \overrightarrow{a_{y}}, \overrightarrow{i_{R}}, \overrightarrow{i_{\theta}}, \overrightarrow{i_{\phi}}$ are unit vectors $\underset{\rightarrow}{\rightarrow}$ cartesian and spherical coordinates respectively, $\vec{m}$ and $\vec{n}$ are equal to $\vec{M}$ and $\vec{N}$, time factors being omitted respectively and $\left[k_{0} R j_{n}\left(k_{0} R_{0}\right)\right]^{\prime}$ means the differentiation with respect to $k_{0} R[2]$.

In course of obtaining the solution of equation (7) we consider $k^{\prime} \rho$ as a perturbation term and seek for a suitable complementary term to be added to the solution (9) of equation (8).

Writing $\phi=f_{1}(R) \cdot f_{2}(\theta) \cdot f_{3}(\phi)$ in equation (7), we get the following equation for $f_{1}(R)$ :

$$
d_{2} f_{1} / d R_{2}+(2 / R)\left(d f_{1} / d R\right)+\left(k_{0}{ }^{2}-n(n+1) / R\right) f_{1}=-k^{\prime} f,
$$

where $n$ is an arbitrary positive integer. Other terms $f_{2}$ and $f_{3}$ satisfy the wellknown differential equations of the case without the perturbation term $k^{\prime} \phi$, and are omitted from the present discussion. In equation (11) we put

$$
f_{1}=g(R) / R \text {, }
$$

and equation (11) becomes

$$
d^{2} g / d R^{2}+\left(k_{0}^{2}-n(n+1) / R^{2}\right) g=-k^{\prime} g .
$$

According to the above-stated consideration we have the solution of equation (12) in the form

$$
g_{n}=R j_{n}\left(k_{0} R\right)+\Phi,
$$

where $\Phi$ results from the perturbation term $k^{\prime} g$. Putting equation (14) into equation (13) and regarding $k^{\prime} \Phi$ to be negligibly small, equation (13) may be written as

$$
d^{2} \Phi / d R^{2}+\left(k_{0}^{2}-n(n+1) / R^{2}\right) \Phi=-k^{\prime} R j_{n}\left(k_{0} R\right) .
$$

Generally the differential equation

$$
d^{2} \Phi / d R^{2}+\left(k_{0}{ }^{2}-n(n+1) / R^{2}\right) \Phi=0,
$$

has two independent solutions $\Phi^{(1)}$ and $\Phi^{(2)}$ representing wave properties. Now we assume $\Phi^{(1)}$ and $\Phi^{(2)}$ to be expressed as 
and

$$
\begin{aligned}
& \Phi^{(1)} \sim \sin \left(k_{0} R+\eta\right) \\
& \Phi^{(2)} \sim k_{0}{ }^{-1} \exp i\left(k_{0} R+\eta\right)
\end{aligned}
$$

for large values of $R$. Then $\Phi^{(1)}$ and $\Phi^{(2)}$ will satisfy, for all values of all $R$,

$$
\Phi^{(2)} d \Phi^{(1)} / d R-\Phi^{(1)} d \Phi^{(2)} / d R=1,
$$

and hence the everywhere-bounded solution of equation (15) is

$$
\Phi=\Phi^{(1)}(R) \int_{\alpha}^{h} \Phi^{(2)}\left\{-k^{\prime} R j_{n}\left(k_{0} R\right)\right\} d R-\Phi^{(2)}(R) \int_{0}^{R} \Phi^{(1)}\left\{-k^{\prime} R j_{n}\left(k_{0} R\right)\right\} d R,
$$

where $\alpha$ is an arbitrary constant [2].

As the solution $\Phi$, expressed by equation (16), results from the nonconductive: inhomogeneity $k^{\prime}$ in the medium, it should behave just like such a travelling wave as $\exp \left(i k_{0} R\right)$ for large values of $R$. Further we consider $k^{\prime}$ to be negligibly small for a large values of $R$ and assume that the integrals in the right hand side of equation (16) converge as $R$ increases indefinitely. Hence the required solution is. obtained by putting $\alpha=\infty$ and is shown in the form (for a large value of $R$ ),

$$
\Phi \simeq-\Phi^{(2)}(R) \int_{0}^{\infty} \Phi^{(1)}\left\{-k^{\prime} R j_{n}\left(k_{0} R\right)\right\} d R
$$

According to their asymptotic forms for large values of $R, \Phi^{(1)}$ may be written. as $k_{0} R j_{n}\left(k_{0} R\right)$ and $\Phi^{(2)}$ as $R h_{n}{ }^{(1)}\left(k_{0} R\right)$ and we get

$$
\left\{\begin{array}{l}
\Phi=A_{n} R h_{n}{ }^{(1)}\left(k_{0}\right) . \\
A_{n}=\int_{0}^{\infty} k_{0} R^{2}\left[j_{n}\left(k_{0} R\right)\right]^{2} k^{\prime} d R .
\end{array}\right.
$$

Therefore, equations (12), (14) and (17) lead to

$$
f_{1 n}=j_{n}\left(k_{0} R\right)+A_{n} h_{n}{ }^{(1)}\left(k_{0} R\right)
$$

and the solution of equation (7) is expressed by

$$
\varphi_{e^{m n}}=\left\{j_{n}\left(k_{0} R\right)+A_{n} h_{n}{ }^{(1)}\left(k_{0} R\right)\right\} P_{n}{ }^{m}(\cos \theta) \sin _{\sin }^{\cos } m \phi e^{-i \omega t}
$$

Consequently $\vec{m}$ and $\vec{n}$, which are obtained respectively from $\vec{M}$ and $\vec{N}$ excluding: the time factors, are obtained from equation (18) in the following form:

$$
\begin{aligned}
& \vec{m}_{o^{m n}}=\vec{m}_{0^{m n}}^{(i)}+A_{n} \vec{m}_{e^{m}}^{(s)}, \\
& \vec{n}_{e_{m n}}=\vec{n}_{e^{m n}}^{(\ell)}+A_{n} \vec{n}_{e^{m n}}^{(s)} \text {, } \\
& \vec{m}_{e_{m n}(i)}=\mp \frac{m}{\sin \theta} j_{n}\left(k_{0} R\right) P_{n}{ }^{m}(\cos \theta) \frac{\sin }{\cos } m \overrightarrow{\phi i_{\theta}}-j_{n}\left(k_{0} R\right) \frac{\partial P_{n}{ }^{m}}{\partial \theta} \cos { }_{\sin } m \overrightarrow{\phi i_{\phi}}, \\
& \left\{\vec{m}_{0^{m n v}}^{(s)}=\left(\text { the same one as replaced } j_{n} \text { by } h_{n n}{ }^{(1)}\right. \text { in the just above equation), }\right. \\
& \vec{n}_{e_{m n}}(i)=\frac{n(n+1)}{k R} j_{n}\left(k_{0} R\right) P_{n}^{m}(\cos \theta) \underset{\sin }{\cos } m \overrightarrow{\phi i_{R}}+\frac{1}{k R}\left[k_{0} R j_{n}\left(k_{0} R\right)\right]^{\prime} \\
& \times \frac{\partial P_{n}{ }^{m}}{\partial \theta} \sin m \phi \overrightarrow{i_{\theta}} \mp \frac{m}{k R \sin \theta}\left[k_{0} R j_{n}\left(k_{0} R\right)\right]^{\prime} P_{n}{ }^{m}(\cos \theta) \underset{\cos }{\sin } m \phi \overrightarrow{i_{\phi}}, \\
& \vec{n}_{0}^{(s)}=\text { (the same one as replaced } j_{n} \text { by } h_{n}(1) \text { in the just above equation', }
\end{aligned}
$$


where $\left[k_{0} R j_{n}\left(k_{0} R\right) \underset{\rightarrow(s)}{\prime}\right.$ means the differentiation with respect to $k_{0} R$. Here it should be noted that $A_{n} \vec{m}$ and $A_{n} \vec{n}$ originate from the inhomogeneity $k^{\prime}$ in the medium.

The required electromagnetic field can now be expanded in terms of the above obtained $\{\vec{m}, \vec{n}\}$ determining the expansion coefficients. If we consider the limiting case when the inhomogeneity in the medium is very small, then $\{\vec{m}, \vec{n}\}$ approaches to $\left\{\vec{m}^{(i)}, \vec{n}^{(l)}\right\}$ and the required field should coincide with the incident plane wave represented by equations (10). This fact demands that the expansion coefficients should be the same as those in equation (10). The required field is then expressed by

$$
\left\{\begin{array}{l}
\vec{E}=\vec{E}^{(i)}+\vec{E}^{(s)}, \\
\vec{H}=\vec{H}^{(i)}+\overrightarrow{H^{(s)}}, \\
\overrightarrow{E^{(i)}}=E_{0} e^{-i \omega t} \sum_{n=1}^{\infty} i_{n} \frac{2 n+1}{n(n+1)}\left(\vec{m}_{01 n}^{(i)}-i \vec{n}_{e 1 n}^{(i)}\right), \\
\vec{E}^{(s)}=E_{0} e^{-i \omega t} \sum_{n=1}^{\infty} i^{n} \frac{2 n+1}{n(n+1)} A_{n}\left(\vec{m}_{e 1 n}-i \vec{n}_{e 1 n}^{(s)}\right), \\
\vec{H}^{(s)}=-\frac{k_{0} E_{0}}{\mu_{0} \omega} e^{-i \omega t} \sum_{n=1}^{\infty} i^{n} \frac{2 n+1}{n(n+1)}\left(\vec{m}_{e 1 n}^{(i)}+i \vec{n}_{01 n}\right), \\
\vec{H}^{(s)}=-\frac{k_{0} E_{0}}{\mu_{0} \omega} e^{-i \omega t} \sum_{n=1}^{\infty} i^{n} \frac{2 n+1}{n(n+1)} A_{n}\left(\vec{m}_{e 1 n}^{(s)}+i \vec{n}_{01 n}^{(s)}\right)
\end{array} .\right.
$$

Now we examine the scattering field denoted by index (s) in equations (19) and (20). At a sufficiently large distance from the center of inhomogeneity, the radial component of the scattering field vanishes as $1 / R^{2}$ does, while the tangential components $E_{\theta}^{(s)}$. and $E_{\phi}^{(s)}$ diminish as $1 / R$ does. This shows that the incident wave is not affected by the inhomogeneity at a sufficiently large distance, and that the scattering field satisfies the so-called radiation conditions. Considering further that the components $E_{\theta}{ }^{(s)}$ and $E_{\phi}^{(s)}$ are perpendicular to each other and differ, in general, in phase, the scattering radiation by the inhomogeneity is said to be "elliptically polarized". There are, however, two exceptions in direction. When $\phi=0$, we note $E_{\phi}^{(s)}=0$, and when $\phi=\pi / 2$ we see $E_{\theta}^{(s)}=0$. The scattering radiation is thus linearly polarized when viewed along the $x$ - or $y$ - axis.

The properties of the scattering field thus far described coincide with those of the scattering field by a sphere.

The Rayleigh scattering, the simplest case of the scattering field by a sphere is the same as the electric dipole field, but the present case is not so simple, as can be seen from the expansion coefficients in equation (20).

\section{Total scattering cross section of the inhomogeneity}

The total scattering cross section is defined here as the ratio of the total scattered energy per unit time to the energy density of the incident wave. 
The total scattering energy flow is shown by

$$
W_{s}=\frac{1}{2} R_{e} \int_{0}^{\pi} \int_{0}^{\Sigma \pi}\left(E_{\theta}^{(s)} \widetilde{H}_{\phi}^{(s)}-E_{\phi}^{(s)} \widetilde{H}_{\theta}^{(s)}\right) R^{2} \sin \theta d \theta d \phi[1] .
$$

The integration for large $R$ leads to

$$
W_{s}=2 \pi \frac{E_{0}{ }^{2}}{k_{0}{ }^{2}} \sqrt{\frac{\varepsilon_{0}}{\mu_{0}}} \sum_{n=1}^{\infty}(2 n+1)\left|A_{n}\right|^{2} .
$$

The mean energy flow of the incident wave per unit area is

$$
S_{z}=\frac{1}{2} E_{0}^{2} \sqrt{\frac{\varepsilon_{0}}{\mu_{0}}} .
$$

Thus the total scattering cross section $Q_{s}$ is

$$
Q_{s}=W_{s}=\frac{4 \pi}{k_{0}{ }^{2}} \sum_{n=1}^{\infty}(2 n+1) A_{n:}^{2} .
$$

\section{An example case}

We shall take such an example as

$$
k^{\prime}=a e^{-R^{2} / R_{0}^{2}} \omega^{2} \text {. }
$$

From equation (17)

$$
A_{n}=\frac{a \pi \omega^{2} R_{0}{ }^{2}}{4} \exp \left(-\frac{k_{0}{ }^{2} R_{0}{ }^{2}}{2}\right) I_{n+1 / 2}\left(\frac{k_{0}{ }^{2} R_{0}{ }^{2}}{2}\right),
$$

where $I_{\nu}$ is the modified Bessel function.

Total scattering cross section is, from equation (23),

$$
\begin{aligned}
Q_{s} & =-\frac{a^{2} \pi^{3} \omega^{4} R_{0}{ }^{4}}{4 k_{0}^{2}}-\exp \left(-k_{0}{ }^{2} R_{0}{ }^{2}\right) \sum_{n=1}^{\infty}(2 n+1)\left[I_{n+\frac{1}{2}}\left(\frac{k_{0}{ }^{2} R_{0}{ }^{2}}{2}\right)\right]^{-1} \\
& \vdots \frac{a^{2} \pi^{3}}{4 \varepsilon_{0}^{2} \mu_{0}{ }^{2}} k_{0}{ }^{2} R_{0}{ }^{4} \exp \left(-k_{0}{ }^{2} R_{0}{ }^{2}\right) \sum_{n=1}^{\infty}(2 n+1)\left[I_{n+\frac{1}{2}}\left(\frac{k_{n}{ }^{2} R_{0}{ }^{2}}{2}\right)^{-2}\right]^{2} .
\end{aligned}
$$

The summation of series in the right hand side of equation (26) can be proved to converge in the following manner.

The integral representation of $I_{2}$, is

$$
I \cdot \nu(z)=\frac{2(1 / 2 z)^{\nu}}{\Gamma(\nu+1 / 2) \Gamma(1 / 2)} \int_{0}^{1}\left(1-t^{2}\right)^{\nu-\frac{1}{2}} \cosh (z t) d t[3] .
$$

It is derived from this representation that

Therefore,

$$
\begin{aligned}
I_{n+\frac{1}{2}}(z) & \leq \frac{2(1 / 2 z)^{n+1}}{\Gamma(n+1) \Gamma(1 / 2)} \int_{0}^{1}\left(1-t^{2}\right)^{n} \frac{\left(e^{|z|}+1\right)}{2} d l \\
& =\frac{\left(e^{i z i}+1\right)}{2} \frac{(1 / 2 z)^{n+\frac{1}{2}}}{\Gamma(n+3 / 2)} .
\end{aligned}
$$

$$
\begin{aligned}
\sum_{n=1}^{\infty}(2 n+1)\left[I_{n+1}{ }_{2}^{1}(z)\right]^{2} \leq \sum_{n=1}^{\infty}(2 n+1) \frac{\left(e^{|z|}+1\right)^{2}}{4} \frac{(1 / 2 z)^{2 n+1}}{[\Gamma(n+3 / 2)]^{-2}} \\
=\frac{\left(e^{|z|}+1\right)^{2}}{4} \sum_{n=1}^{\infty} \frac{(2 n+1)(z / 2)^{2 n+1}}{[\Gamma(n+3 / 2)]^{2}}
\end{aligned}
$$

The right hand side of this inequality is obviously summable. Consequently the 
left hand side of the inequality, that is, the right hand side of equation (26) converges.

When the region, or spatial magnitude, $R_{0}$ of inhomogeneity is so small that $I_{n+1 / 2}\left(k_{0}{ }^{2} R_{0}^{2} / 2\right)$ is sufficiently expressed only by the first term of the power series expansion of $\left(k_{0}^{2} R_{0}^{2} / 2\right)$,

$$
Q_{s}=\frac{a^{2} \pi^{3} k_{0}{ }^{2} R_{0}{ }^{4}}{4 \varepsilon_{0}{ }^{2} \mu_{0}{ }^{2}} \exp \left(-k_{0}{ }^{2} R_{0}{ }^{2}\right) \sum_{n=1}^{\infty} \frac{(2 n+1)}{\left[\Gamma\left(n+{ }^{3} / 2\right)\right]^{2}}\left(\frac{k_{0}{ }^{2} R_{0}{ }^{2}}{4}\right)^{2 n+1} .
$$

If $R_{0}$ is further small so that the main part may be the first term in the summation of the right hand of equation. (27),

$$
Q_{s}=\frac{a^{2} \pi^{2} k_{0}{ }^{8} R_{0}{ }^{10}}{48 \varepsilon_{0}^{2} \mu_{0}^{2}} \exp \left(-k_{0}{ }^{2} R_{0}{ }^{2}\right)
$$

From equation (26), the total scattering cross section $Q_{s}$ is proportional to the squares of qualitative magnitude $a$ of inhomogeneity.

Acknowledgement _... The present author wishes to express his gratitude to Dr. M. SAnuki and Mr. O. Tsukiji for their kind advices.

\section{References}

[1] Srratron, J. A., 1941: Electromagnetic Theory, New York, McGraw-Hill.

[2] Mott, N. F. \& Massey, H. S. W., 1949: The Theory of Atomic Collisions, London, Oxford University Press. pp. 106-108.

[3] Watson, G. N., 1922: Theory of Bessel Functions, Cambridge University Press, p. 79. 